\title{
LES CARPES CHINOISES
}

\author{
Par P. CHIMITS \\ Ingénieur des Eaux et Forets.
}

Le numéro du Journal Officiel du 28 Mai 1957 contient (p. 5347) l'arrêté ministériel ci-dessous :

"Autorisation d'acclimation de poissons herbivores subtropicaux dans les eaux libres du territoire français. - Par décision du 16 Mai 1957, M. l'Ingénieur des Eaux et Forêts Снгмiтs à Pau, est autorisé sous le contrôle de la Station d'Hydrobiologie appliquée, à procéder à des essais d'acclimatation des deux espèces de poissons herbivores subtropicaux suivants :

a) La Carpe chinoise (Ctenopharyngodon idellus);

b) Le Tilapia sparmani.

On peut se demander à quels besoins pour nos eaux piscicoles françaises correspond l'introduction de ces deux espèces en France, alors qu'en général les introductions d'espèces nouvelles ont mauvaise presse depuis l'arrivée de la Perche-soleil et du Poisson-chat. Ces deux précédents fâcheux (bien que pour la Perche-soleil les inconvénients aient été fortement grossis et d'ailleurs cette espèce est plutôt en voie de régression en France) ont donné jour à une méfiance systématique envers les introductions d'espèces exotiques alors qu'elles sont couramment employées dans d'autres domaines, en matière de sylviculture par exemple.

Il faut distinguer entre les introductions dues à des amateurs aussi bénévoles et enthousiastes que peu compétents qui ont ouvert imprudemment la boîte de Pandore et les acclimatations raisonnées d'espèces correspondant à un besoin, d'une part et, en tout cas, peu susceptibles d'envahir nos eaux de façon abusive.

Or, Carpe chinoise et Tilapia sparmani répondent à ces deux conditions. La Carpe chinoise, d'une part, ne peut se reproduire en étang et sa reproduction, même artificielle, bien que tentée dans divers pays et notamment en Israël, n'a jamais pu, jusqu'à ce jour, avoir lieu ailleurs que dans son aire d'origine : la Chine centrale et méridionale et le Tonkin. Quant au Tilapia sparmani, il est tué par des températures hivernales 
abaissant l'eau à 7 ou $8^{\circ}$, ce qui restreint son aire possible d'acclimatation à l'extrême Sud-Ouest et à la zone méditerraéenne. Il n'y a donc pas de danger de ce côté.

Quelle est donc la raison pour laquelle cette introduction serait intéressante dans nos eaux françaises ? C'est qu'il s'agit, dans les deux cas, d'espèces herbivores, susceptibles de coloniser ce que l'on appelle en langage biologique " une niche écologique vide ", c'est-à-dire, si l'on veut, une nourriture naturelle produite par nos eaux et actuellement inutilisée par notre faune piscicole. Or, nous n'avons pas, en France, de poissons herbivores purs. Nous avons des mangeurs d'algues filamenteuses, tels que le Hotu, la Vandoise, le Muge et, à un plus faible degré, le Gardon blanc et la Tanche ; mais, pratiquement, nous n'avons aucun mangeur de végétation supérieure à part le Gardon et la Tanche qui, au printemps, broutent les jeunes pousses de myriophylles et de potamots. Au contraire, Tilapia sparmani et Carpe chinoise sont des herbivores purs, qui se nourrissent presque exclusivement de végétation supérieure. Le Tilapia se nourrit de lentilles d'eau, de sagittaires, de charas, de nitellas et mème d'Elodea canadensis, cet Élodea si répandu dans nos eaux et si envahissant qu'on l'a surnommé " la peste des eaux douces ". Quant à la Carpe chinoise, nous verrons plus loin sa prodigieuse capacité digestive. Il y a donc dans nos eaux françaises une niche écologique vide, une large place pour des poissons herbivores purs et un intérêt double, d'une part à voir la végétation excessive disparaître sans frais et sans effort humain, par le faucardage par exemple, et d'autre part, à voir cette végétation transformée en poisson produit gratuitement, pratiquement sans emprunt de nourriture aux espèces locales. Il s'agirait donc d'une production supplémentaire gratuite de poissons se superposant à la production naturelle actuelle.

Je reviendrai plus tard sur le Tilapia sparmani : ce poisson qui reste de petite taille est un excellent poisson-fourrage pour les voraces tels que Brochets, Perches et Black-bass. Je me contenterai aujourd'hui de remercier ici bien vivement le Department of Nature Conservation d'Afrique du Sud et son aimable Directeur de la Station de Stellenbosch, qui nous a gracieusement envoyé 24 alevins de Tilapia sparmani par avion, arrivés vivants à Paris fin Juillet 1957.

A la suite de l'arrêté ministériel indiqué ci-dessus, j'ai pu également déclencher l'opération Carpe chinoise grâce au dévouement et à la parfaite compréhension de M. Yu-Y Département d'Agriculture Forêts et Pêche de Hong-Kong et qui, d'Octobre à Décembre 1955, fùt élève du Centre de perfectionnement des Pêches intérieures de Bogor à Java (Indonésie) où j'étais professeur. Ce Centre de perfectionnement avait été fondé par l'Organisation des Nations-Unies pour l'Alimentation et l'Agriculture, à l'usage des fonctionnaires supérieurs des Pêches intérieures des pays d'Extrême-Orient. C'est auprès de lui et de M. Shao-Wen-Ling, expert des Pêches à la F. A. O. et également professeur à ce Centre, que j'ai pu recueillir bon nombre de renseignements pratiques sur la biologie et l'élevage des 
Carpes chinoises pratiqué en Chine et en dehors de la Chine, dans les pays d'Extrême-Orient où réside une forte colonie chinoise. C'est ainsi que j'ai pu voir des élevages de Carpes chinoises en étang de pisciculture notamment à Java, en 'Thaïlande et fen:Bírmanie, élevages également pratiqués dans toute la Malaisie.

Les 110 alevins de Carpe chinoise arrivés sans perte à Orly le 3 Juillet 1957 ont été pondus dans le Yang-Tsé en Mai 1957. Capturés à l'état d'alevins minuscules par des paysans chinois et transportés sur de longues distances sur l'épaule de coolies jusqu'à Hong-Kong, ils ont alors été mis dans un petit récipient métallique étanche avec une provision d'oxygène sous pression, puis placés dans l'avion d'Air-France quittant Hong-Kong le 1 er Juillet pour arriver à Paris le 3 Juillet. Après quelques jours de repos dans un bassin de la Station Centrale d'Hydrobiologie appliquée, le garde-pèche Daurat vint nous en apporter une soixantaine par chemin de fer jusqu'à Dax et ils furent alors placés dans un petit bassin en terre de 4 mètres sur 10 mètres. Du 6 Juillet au 6 Octobre, et malgré un été peu ensoleillé et plutôt froid, ces alevins passèrent d'un poids de 1 gramme à un poids de 15 à 20 grammes, se nourrissant d'algues vertes et de lentilles d'eau. La lentille d'eau est, en effet, un des aliments préférés de la jeune Carpe chinoise.

\section{LES RÉGIMES ALIMENTAIRES DES CARPES CHINOISES}

Revenons à la pisciculture de la Carpe en Extrême-Orient. La Carpe commune (Cyprinus carpio) y est cultivée un peu partout en Inde, Thaïlande, Viet-Nam, Chine, Malaisie et Indonésie. Dans ces pays d'Extrême-Orient, existent deux grands groupes d'espèces de Carpes qui font l'objet d'une pisciculture particulièrement importante. C'est, d'un côté, le groupe des grandes Carpes indiennes auquel se rattachent les genres Catla, Cirrhina et Labeo, largement cultivés dans l'Inde, le Pakistan et, à un moindre degré, en Birmanie. Puis le groupe des Carpes chinoises qui compte également toute une série d'espèces assez variées et de régimes alimentaires très différents, particularité qui leur permet d'être utilisées au mieux en pisciculture.

La plus connue de ces Carpes est précisément celle qui vient d'être importée, le Ctenopharyngodon idellus. C'est la Carpe mangeuse d'herbe, originaire du centre de la Chine et notamment du bassin du Yang-Tsé. Elle mange à peu près toutes les espèces de plantes aquatiques immergées. Elle vient même brouter les herbes terrestres poussant au bord des étangs, y compris le paspalum ou chiendent. Comme elle a vite fait de nettoyer de toute végétation les étangs où elle est placée (et je l'ai particulièrement constaté dans les piscicultures thaillandaises), on la nourrit artificiellement avec des feuilles d'arbres, notamment de bananiers, des herbes fauchées, mais également du fumier, du tourteau et des cocons de vers à soie. J'ai vu, près de Bangkok, des pisciculteurs ramasser des paniers de lentilles d'eau dans les canaux pour les apporter à leurs Carpes. Il arrive même 
que les gros sujets avalent accidentellement des morceaux de vêtements et de vieilles chaussures pourries. Aussi, en Extrême-Orient, grossit-elle vite et atteint-elle 500 grammes à 1 an, 2 kgs à 2 ans, pour grossir ensuite régulièrement d'un kilo par an. Elle atteint couramment une douzaine de kilos et peut aller jusqu'à 20 kilos.

Une autre Carpe chinoise est le Mylopharyngodon piceus, ou Carpe noire. Elle grossit aussi vite que la précédente. C'est une mangeuse de mollusques aquatiques dont elle broie sans difficulté les coquilles.

Une troisième espèce de Carpe est l'Aristichthys nobilis ou Carpe à grosse tête qui est planctonophage.

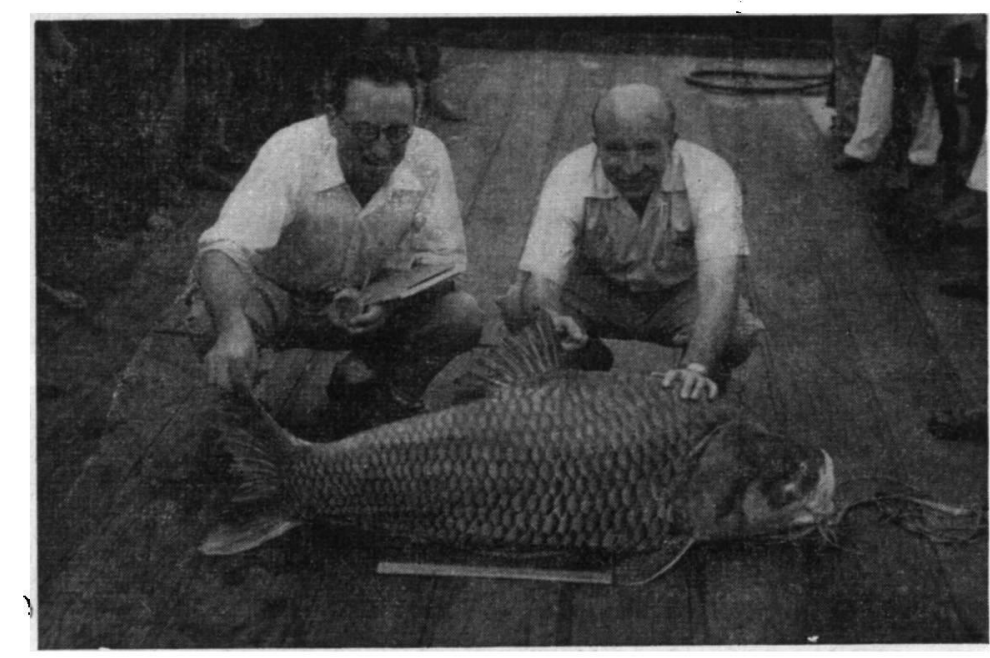

Catla carpio siamensis

Cliche HunT

Poisson du groupe des grandes carpes indiennes, capturé dans la MENAM, à BANGKOK, en octobre 1955. Poids : $46 \mathrm{kgs}$.

Une quatrième espèce est l'Hypophtalmychtis molitrix ou Carpe argentée qui est une mangeuse d'algues, mais aussi de détritus et de végétation supérieure en décomposition.

Une cinquième espèce est la Cirrhina molitorella ou Carpe de vase qui se nourrit sur le fond des étangs, mangeant les vers et larves aquatiques qui vivent dans la vase, mais aussi tous les détritus animaux et végétaux de fond.

Ces cinq espèces sont toutes originaires de Chine centrale ou de Chine du Sud et du bassin du Yang-Tsé. Toutes vivent à l'état sauvage en rivière et ne peuvent se reproduire qu'en rivière. Aucune reproduction n'a pu être obtenue en étang, bien qu'elles soient cultivées en ExtrêmeOrient depuis des millénaires. Les Chinois, qui pratiquent la pisciculture en étang de ces diverses espèces depuis si longtemps, connaissent fort bien les différences de régime alimentaire des diverses espèces; aussi, dosent-ils savamment la charge en alevins des diverses espèces selon la 
richesse de leurs étangs en diverses nourritures naturelles et selon les facilités d'approvisionnement en diverses nourritures artificielles. Une charge classique d'étang chinois se composera par exemple de :

- 40\% d'herbivores purs (Ctenopharyngodon idellus);

- 20\% de planctonophages (Aristichtys nobilis);

- $10 \%$ de mangeurs de mollusques (Mylopharyngodon piceus);

- $30 \%$ d'omnivores mangeant sur le fond et mangeurs de détritus (Hypophtalmichtys molitrix et Cirrhina molitorella).

Ainsi toute la productivité naturelle de l'étang est-elle utilisée. En outre, s'y ajoute le bénéfice des fumures par les excréments de chaque espèce. Les excréments de la Carpe mangeuse d'herbes développe le plancton flottant et le benthos, c'est-à-dire la faune et la flore du fond. Les excréments de la Carpe planctonophage à leur tour développeront la faune et la flore du fond. La Carpe mangeuse de benthos ainsi que les Carpes mangeuses de mollusques et détritus en bénéficieront finalement Ainsi la végétation aquatique sert-elle à produire du poisson, directement et indirectement.

Dans l'Inde et au Pakistan des charges analogues faites avec les diverses espèces de Carpes indiennes sont également utilisées.

Évidemment, les pourcentages des diverses espèces varient aussi suivant la nourriture artificielle que l'on peut se procurer. Voici par exemple une autre combinaison chinoise, que l'on peut qualifier de "combinat "piscicole et industriel. Près des villes chinoises où se trouvent des industries de la soie, il existe dans la banlieue de très nombreux étangs à Carpes où l'on élève la Cténopharyngodon, mangeuse d'herbe mais se nourrissant aussi de cocons de vers à soie. Les bords et les digues de ces étangs sont plantés de mûriers dont les feuilles nourrissent les vers à soie; les produits des dévasages périodiques des étangs sont reportés sur les berges et fument la terre, favorisant la pousse du mûrier et de ses feuilles. Après dévidage de la soie, les cocons sont jetés dans les étangs pour nourrir les Carpes. On voit, par ces quelques exemples, l'habileté et le perfectionnement technique des pisciculteurs chinois. Je pourrais en citer bien d'autres exemples sur d'autres poissons, mais, tout en restant dans le seul sujet des Carpes chinoises, nous allons voir maintenant comment les Chinois s'y prennent pour capturer, trier et compter leurs alevins.

\section{CAPTURE, TRIAGE ET COMPTAGE}

\section{DES DIVERSES ESPECES D'ALEVINS DE CARPES CHINOISES}

Nous avons vu qu'aucune de ces Carpes chinoises ne peut se reproduire en étang et que tous les alevins proviennent de ramassages faits dans les rivières, et notamment le Yang-Tsé, à la partie supérieure desquelles ces Carpes émigrent pour pondre. Une importante et millénaire industrie de pêche de ces alevins existe en Chine de même qu'en Inde 
et au Pakistan pour les grandes Carpes indiennes. D'après S. W. LiNg, cette industrie occupe, à la saison, un grand nombre de pêcheurs expérimentés encadrés par des maîtres-pêcheurs et qui collectent, par an, environ 15 milliards d'alevins de Carpes chinoises. Ces alevins, malgré leur très petite taille (ils sont capturés à l'état de larves de quelques jours et vendus quelques jours plus tard) font l'objet d'un très important commerce intérieur et aussi extérieur, ce dernier par l'intermédiaire de Hong-Kong d'où ils sont expédiés, généralement par avion, dans des récipients clos, sous pression d'oxygène.

Le ramassage a lieu à des périodes bien déterminées et dans des endroits bien précis, à l'aide de poches à mailles très fines. Les alevins innombrables, de très petite taille et transparents, sont à peine visibles, Ils sont rapidement placés dans des récipients en toile flottant dans l'eau. Personnellement, je n'ai pas vu cette opération, mais j'ai vu opérer, dans des conditions analogues, la capture en mer des alevins de chanos, sur la côte Est de Java, selon des techniques minutieuses introduites par les Chinois. Il est presque incroyable, pour des pisciculteurs occidentaux, de voir comment ces minuscules larves peuvent être capturées, transvasées de récipients en récipients et transportées à dos d'homme ou sur des véhicules pendant 10 ou 15 jours, sans pratiquement subir de pertes.

La collecte faite, reste maintenant à trier les diverses espèces de Carpes. Or, les alevins sont à peine discernables à l'œil nu et, même au microscope, il est difficile de déterminer les espèces. Les Chinois ont cependant trouvé une astucieuse méthode pour les séparer. On commence d'abord par enlever les alevins des espèces autres que les Carpes. Cela est assez facile car il s'agit d'alevins de prédateurs nés un peu avant les Carpes et, par conséquent, plus gros. Il suffit de passer, à la partie supérieure des récipients, des épuisettes à mailles fines d'un diamètre déterminé. Les alevins de Carpes se ramassent, en effet, en bancs massifs dans le fond, les alevins prédateurs, plus gros, tournent autour de ces bancs, dans la partie supérieure des récipients. Reste à séparer les alevins des 5 espèces de Carpes. On emploie alors des récipients spéciaux en forme de grandes jarres où on les laisse en repos quelques minutes. Dès lors, les alevins se groupent d'eux-mêmes par espèces et chaque espèce se stratifie dans le récipient soit pour des raisons de tropismes physicochimiques et biologiques (besoin d'oxygène, lumière, pression d'eau), soit peut-être pour des raisons psychiques (instinct grégaire). C'est ainsi que la Carpe argentée formera la couche supérieure, la Carpe à grosse tête la couche suivante et la Carpe mangeuse d'herbe avec la Carpe mangeuse d'escargots occuperont les deux couches qui viennent ensuite, tandis que la couche la plus basse sera constituée uniquement par des Carpes mangeuses de vase. Adroitement, les coups d'épuisettes à mailles très fines captureront les alevins par couches bien délimitées et les placeront dans des récipients séparés. L'opération, dans chaque récipient, sera renouvelée deux ou trois fois et, à la troisième reprise, les alevins sont pratiquement tous de la même espèce et sont dès lors prêts à la vente et au transport.

Quant au comptage des alevins, il est fait par unité au stade du détaillant, exactement comme je l'ai vu faire en Indonésie pour les alevins 
de chanos. Mais, au stade du collecteur et de la vente en gros, cela serait impossible et les Chinois ont encore une méthode astucieuse qui leur permet une estimation très précise du nombre de leurs alevins. La masse d'alevins à compter est placée dans un récipient d'environ $1 \mathrm{~m} . \times 2 \mathrm{~m}$. à fond de toile très fine. Au bout de quelques minutes, la répartition des alevins dans le récipient est devenue homogène. Grâce à un bâton qui coulisse verticalement, exactement dans le milieu du récipient, on lève la toile du fond et le récipient est ainsi partagé en deux parties égales. L'une des deux moitiés est vidée de son contenu et ses alevins

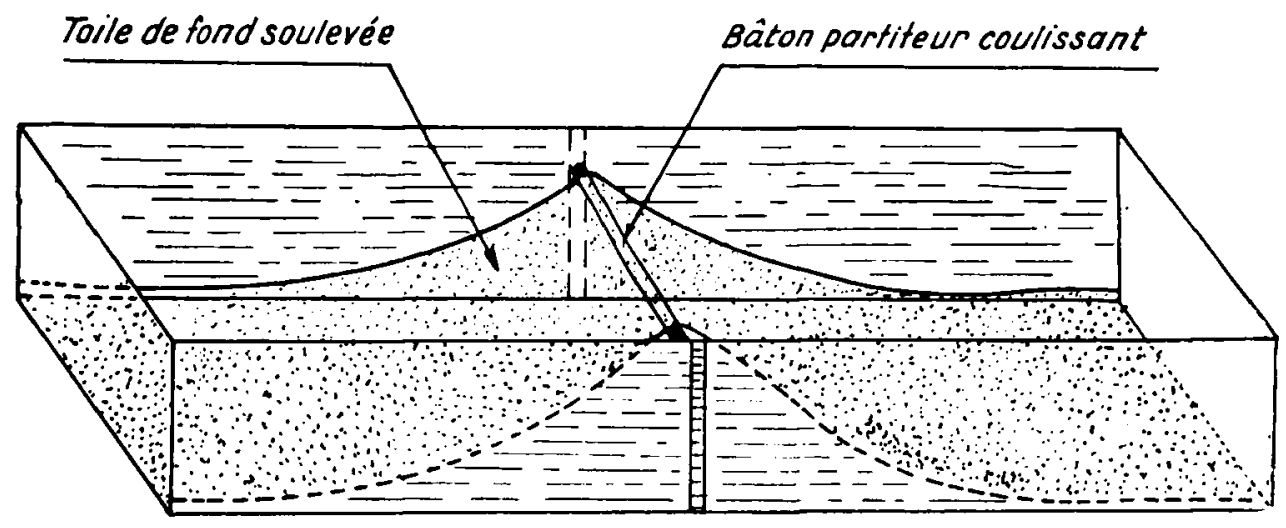

Cuve chinoise de comptage d'alevins.

sont placés dans un autre récipient; on abaisse alors le bâton du récipient de comptage. Les alevins restants, qui sont la moitié du total, se répartissent à nouveau dans l'ensemble du récipient et, l'homogénéité obtenue au bout de quelques minutes, on recommence l'opération. Au bout d'une série de tels comptages, on n'a plus à compter qu'un petit nombre d'alevins. Il suffit de multiplier le nombre trouvé par 32 si on a fait cinq opérations, par 64 si on en a fait six, par 1.024 si on en a fait dix, par 2.048 si on en a fait 11, etc. Technique d'échantillonnage connue en Occident pour les numérations biologiques mais qui, du côté chinois, a le mérite d'être extrêmement ancienne et de s'appliquer à de minuscules alevins qu'il faut garder vivants. Dans la méthode européenne d'élevage des Carpes en frayères Dubish, nous avons employé une méthode d'échantillonnage à peu près semblable pour compter les alevins de Carpes (voir le Bulletin Français de Pisciculture, nos 142 et 144), mais il faut reconnaître que cette méthode chinoise est nettement plus astucieuse. 


\section{CONCI.USION}

Nos 60 alevins de Carpes chinoises se trouvent donc actuellement dans un bassin de la pisciculture du Rancez, près de Dax. Espérons qu'ils pourront passer l'hiver sans inconvénient et que nous pourrons étudier leur comportement et leur régime alimentaire dans les conditions locales.

Pour le moment, étant donné que la Carpe chinoise ne peut se reproduire en étang, son avenir en France semble très limité et je ne la vois guère utilisée qu'en qualité de "fonctionnaire municipal " chargé de débarrasser d'algues, lentilles d'eau et d'herbes aquatiques les pieces d'eau ornementales des parcs et jardins publics, rôle non négligeable et susceptible de faire économiser des frais d'entretien et de faucardage assez onéreux. Mais, la Carpe chinoise constituera un matériel idéal pour nos laboratoires de physiologie piscicole, pour tenter de mettre au point une méthode de mûrissement artificiel du poisson tel que l'hypophysation (1).

Si on arrive à obtenir, par cette technique, le mûrissement artificiel de la Carpe chinoise, la fécondation artificielle à sec serait dès lors relativement facile; elle a d'ailleurs été réalisée en Chine à partir de reproducteurs déjà mûrs. Si on arrive un jour à provoquer un mûrissement artificiel des géniteurs de Carpe chinoise, alors s'ouvrira pour elle et pour la production piscicole de nos eaux françaises un très bel avenir : entretien et faucardage gratuits de nos étangs et rivières lentes, production supplémentaire de poissons sans inconvénients et même, au contraire, avec avantages pour la production normale des poissons indigènes et aucun danger d'envahissement puisque la reproduction de cette Carpe resterait entièrement contrôlée par l'homme.

Mais cette perspective n'est pas pour aujourd'hui. Contentonsnous maintenant d'observer chez nous les quelques spécimens de Carpes chinoises que nous avons pu importer.

Je terminerai en indiquant une autre raison à l'essai d'introduction d'espèces tropicales. C'est qu'en effet, dans un proche avenir, nous risquons en France un fort réchauffement de certaines eaux, à l'aval de certaines puissantes usines thermiques ou atomiques. Un cas précis dans le Sud-Ouest est celui de la future énorme usine thermique de Lacq qui réchauffera les eaux du Gave de Pau d'une dizaine de degrés. Les espèces locales risquent de n'y point survivre et l'utilisation d'espèces tropicales constituerait une solution élégante à ce problème.

(1) Il est à noter que cette technique d'hypophysation n'offre pas d'intérèt pratique pour les espèces à reproduction normale. 